\title{
Tuft deflection velocimetry: a simple method to extract quantitative flow field information
}

\author{
Ben Steinfurth $^{1}$ (I) C. Cura ${ }^{1} \cdot$ J. Gehring ${ }^{1} \cdot$ J. Weiss $^{1}$
}

Received: 10 February 2020 / Revised: 12 May 2020 / Accepted: 15 May 2020 / Published online: 6 June 2020

(c) The Author(s) 2020

\begin{abstract}
A novel method capable of assessing flow fields in a quick and relatively simple manner is introduced. In an extension to the classical qualitative flow visualization by means of cotton or polymeric tufts, digital data processing is used to extract the orientation of these tufts. This information can be related to physical quantities, in particular to time- and space-dependent velocity signals. The capability of this method is demonstrated in two test scenarios. First, it is applied to gain information on the unsteady near-wall flow along a turbulent separation bubble. Second, the two-component velocity field in the wake of a generic car model is measured, allowing for a quantification of the recirculation zone dimensions. Validation measurements with conventional techniques, e.g., particle image velocimetry, unsteady pressure measurements and hot wire anemometry, are conducted throughout the study. These generally suggest that the novel approach provides a quick and reasonably good quantitative overview of the flow configurations. However, the measurement error may be substantial in flow regions of low velocity or dominated by high-frequency oscillations.
\end{abstract}

\section{Graphic abstract}

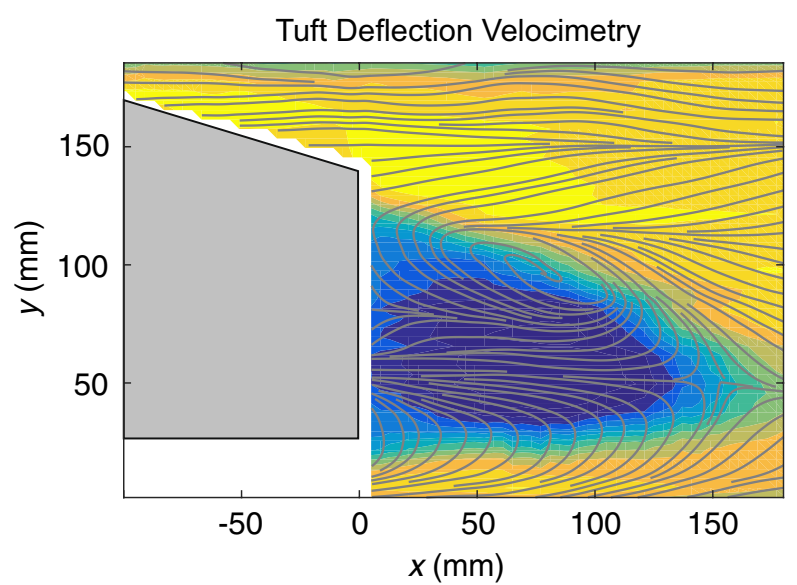

Electronic supplementary material The online version of this article (https://doi.org/10.1007/s00348-020-02979-7) contains supplementary material, which is available to authorized users.

Ben Steinfurth

ben.steinfurth@tu-berlin.de

J. Weiss

julien.weiss@tu-berlin.de

1 Chair of Aerodynamics, Technische Universität Berlin, Berlin, Germany

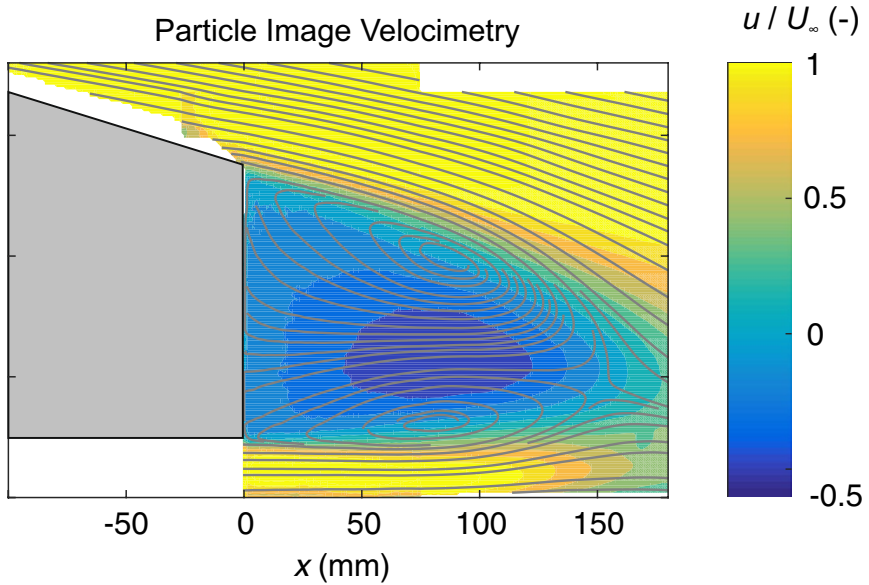

\section{Introduction}

For centuries, visualizing fluid flow phenomena has played a crucial role in understanding the underlying physical mechanisms. This is due to the fact that " $[\ldots]$ once a phenomenon is visualized, a large step has been taken toward understanding it and toward solving the theoretical and experimental problems involved" (Werle 1973). For example, the visualization experiments of Reynolds (1883) and Mach (1887) 
contributed significantly to the understanding of fundamental flow physics while more recently, the discovery of coherent structures was enabled by flow visualization methods (Brown and Roshko 1974).

As suggested by Ristić (2007), flow visualization methods can be classified into surface flow and off-the surface flow visualization, depending on the flow region that is analyzed. Surface flow visualization is primarily used to obtain information regarding the boundary layer state, e.g., the transition point, the local direction of wall shear stress and the lines of separation and reattachment. Along with oil film visualization, a qualitative analysis with surface tufts is commonly performed. The model is hereby equipped with thin fabric tufts that are glued to its surface. The orientation of individual tufts is then governed by the space-dependent velocity field, and intuitive conclusions regarding the flow field can be drawn. A short and incomplete list of applications includes airplane wings and components, wind turbines, road vehicles and race cars (Andino et al. 2015; Eggleston and Starcher 1990; Fisher et al. 1991; Steinfurth et al. 2019). Off-the surface flow visualization techniques are used to visualize characteristic flow features outside the boundary layer by indicating streamlines. This can be achieved by using smoke or oil droplets in air and dye/ink in water. However, tufts may be employed in this scenario as well by arranging them in a gridwise manner, e.g., attached to a screen. For example, placing this screen at various positions in the flow enabled investigations of the longitudinal vortices in the wake of a NACA 0012 wing (Mason and Marchman III 1971), semiwing models (McCormick et al. 1968), rectangular wings and delta wings (Bird and Riley 1952).

Previous studies have shown that there is a large bandwidth of possible operation scenarios for the classical tuft visualization technique-both in surface and off-surface application. This is no doubt due to the intuitive function principle of the approach as the free ends of the tufts mimic the major flow characteristics. Qualitative statements about the flow can then readily be made since the tuft deflection is directly linked to local velocity vectors through the flow momentum.

The desire to extend this conventional approach and gain quantitative information regarding velocity fields is therefore natural. Such a development, from a qualitative to a quantitative method, is by no means new as for instance, particle image velocimetry has evolved from flow visualizations with smoke particles, benefited by advancing imaging and data processing methods.

Thus, a few recent studies were aimed at gaining quantitative insight from images of tuft visualization. Vey et al. (2014) analyze the stall behavior of full-scale wind turbines using per-tuft statistics as the indicator of the local flow. They link the tuft angular standard deviation to the local turbulence level which allows for the determination of separation lines. Wieser et al. apply a similar methodology to a realistic car model (Wieser et al. 2015) and full-scale road vehicles (Wieser et al. 2016). Here, mean tuft angles are also used to perform line integral convolution (LIC). Their results show good agreement between the calculated surface traces and oil film visualization as well as CFD simulations (Wieser et al. 2016). Swytink-Binnema and Johnson (2016) determine the blade stall from captured videos of the tufts applied to a wind turbine blade. Using luminescent minitufts, Chen et al. conduct a quantitative analysis of the tuft inclination angle on a flat plate (Chen et al. 2019) and a backward-facing step (Chen et al. 2020).

While all aforementioned studies aimed at extending the classical tuft visualization technique to extract quantitative flow field information, a method that can be easily adapted by other researchers has not been presented to the best of our knowledge. Furthermore, there appear to be no attempts to relate the tuft deflection to the momentum of the flow and thus allow for the measurement of the flow velocity.

The objective of this paper is therefore to introduce a method that is easily adaptable by other research groups and allows to extract quantitative velocity data from captured tuft images without the need for commercial software. The basic principle is demonstrated by means of two examples, representing surface and off-surface applications. First, unsteady near-wall quantities are obtained in a turbulent separation bubble (TSB) by affixing tufts to the wall in a one-sided diffuser. Then, the turbulent wake of an Ahmed body is investigated by means of a traversable probe with a large number of tufts.

\section{Evaluation routine}

The basic evaluation routine for tuft deflection velocimetry (TDV) is exemplarily displayed in Fig. 1. Note that a minimum working example of the algorithm is also provided with the supplementary material. Following the application of tufts inside a given flow field, the first step in obtaining quantitative information consists in the recording of images. A priori, the method is not limited to coherent snapshots but the acquisition rate of the camera must be adjusted to the time scales associated with relevant unsteady flow features in order to study the dynamic flow behavior. Sufficient lighting is required so that tufts are captured in their entirety. This can be particularly challenging in the case of high acquisition rates and low exposure times. In practice, tufts of UV active material illuminated with appropriate LED arrays are successfully employed in this scenario. Based on recorded images, the geometries of individual tufts can be extracted. Since they are typically very thin compared to their length, the tuft edges can be considered a satisfying representation of the tuft deflection. To extract these edges, the intensity gradient field is evaluated. While there 

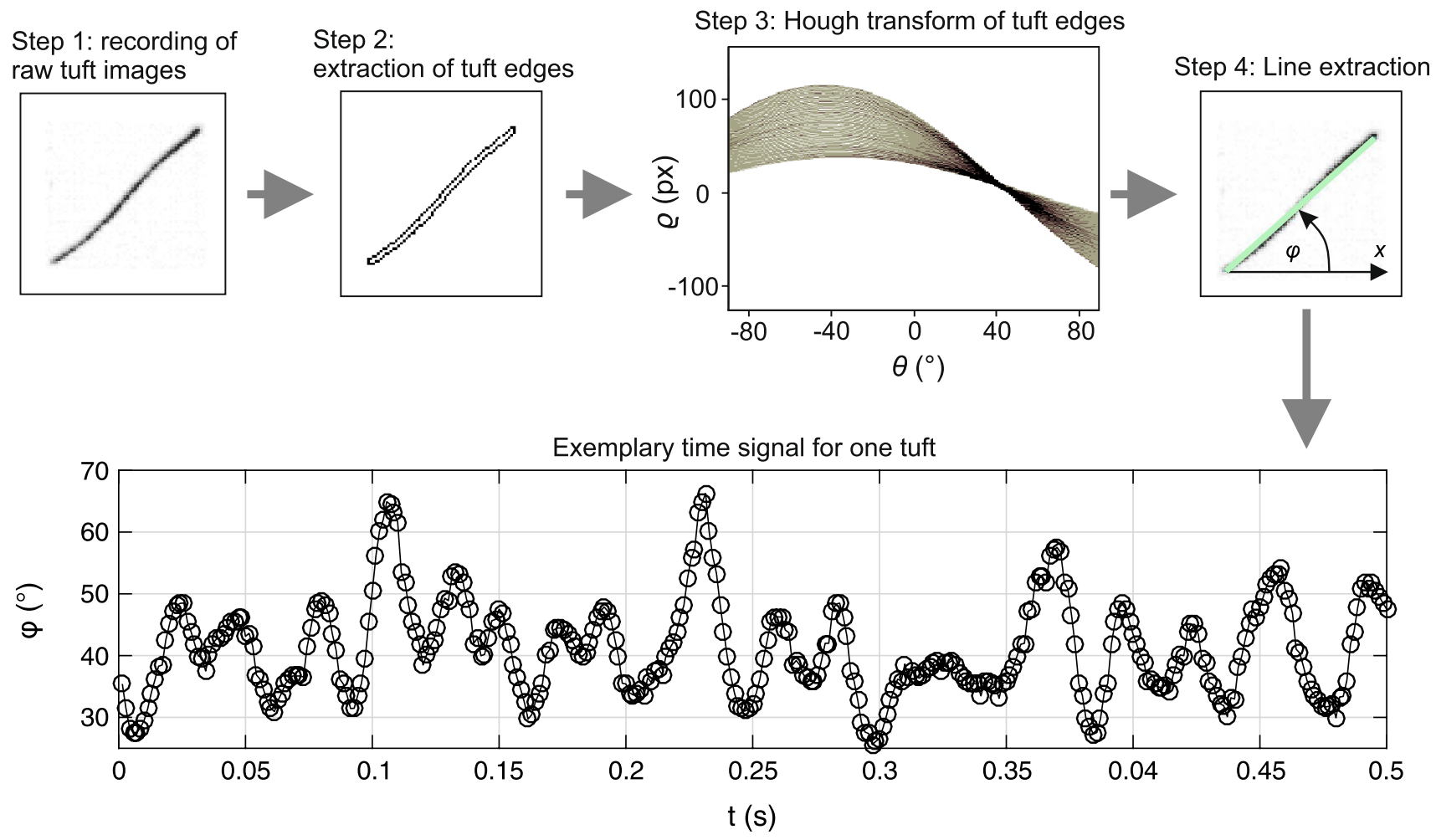

Fig. 1 Flow diagram visualizing the evaluation of the deflection angle of one tuft

are several different approaches, we found the Prewitt method (1970) to be the most accurate and cost-efficient for the demonstration test cases addressed in the following.

The Prewitt operator employs two $3 \times 3$ kernels that are convolved with an image to approximate the intensity derivatives in horizontal and vertical direction, respectively. This separable approach makes it efficient but may also cause inaccuracies when dealing with high-frequency variations in the intensity function. Therefore, a sufficient contrast needs to be ensured between tufts and background. In experiments, this is typically achieved by applying a black foil or coating to the background if required. The Prewitt filter then returns a zerogradient for such a background whereas the interface between tuft and background is detected as an edge and will be returned in a binary image (step 2 in Fig. 1). This binary image then undergoes a Hough transformation (step 3), a robust standard procedure to extract parametrizable geometries (Duda and Hart 1972). In the case of line extraction required for TDV, the Hough space is spanned by the Euclidean distance of the line $\rho$ and the angle between the perpendicular of the line and the abscissa $\vartheta$. Thus, the line can be represented by its Hesse normal form

$\varrho=x \cos \vartheta+y \sin \vartheta$.
It is worth noting that the angle given in the Hesse normal form already is an indicator for the tuft orientation. However, $\vartheta$ depends on the dimensions of raw images since the location of the abscissa with respect to the tuft/ extracted line may be varied. Therefore, we employed a tracking method to detect the end points of the parametrized lines (step 4) and computed the tuft deflection angle based on their locations. For certain applications such as the second demonstration example in this article, it is also necessary to compute the length of the detected lines which inherently requires knowledge regarding their end points as well.

Repeating this procedure for multiple successive images, the time-dependent deflection angle of tufts can be derived for further statistical analysis of a given flow field.

\section{Choosing tufts with appropriate properties}

The accuracy of TDV is directly dependent on the characteristics of the employed tufts. A brief summary of the main parameters that need to be considered for tuft selection is given in this section. 


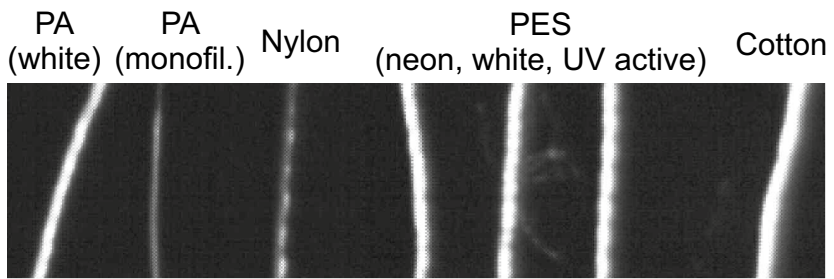

Fig. 2 Visibility of different tuft materials of similar diameter for equal lighting conditions

\subsection{Tuft visibility}

Being an optical measurement technique, good visibility of the tufts is required, ensuring that the tuft geometry is adequately represented in pixel space. For a given lighting setup, two parameters majorly affect the visibility: (a) the tuft thickness and (b) its color/reflectivity.

To reduce the influence on the flow field, tufts of minimum dimensions are preferred. This minimum extent strongly depends on the optical resolution of the measurement setup, i.e., the size of the measurement domain and the camera chip. A low resolution can be compensated by a bright tuft color which is exemplarily demonstrated in Fig. 2 where tufts of different materials but similar diameters are shown. Whereas the images of transparent tufts of polyamid (PA) monofilament and nylon are associated with low contrast, the other tufts clearly reflect a greater amount of light and are therefore better suited for the measurement technique.

\subsection{Static behavior of tufts}

For a specific flow momentum, the deflection angle of a tuft is determined by its length and stiffness. Again, longer tufts are not desired due to the greater influence on the flow field and because the velocity information is integrated across greater spatial scales. On the other hand, short tufts are typically not suited for low-speed measurements as the deflection angle is small compared to the measurement accuracy in this case.

The characteristic deflection curves for tufts of length $l=10 \mathrm{~mm}$ are shown in Fig. 3. To compile these curves, a nozzle was supplied with a constant mass flow rate, resulting in a steady flow approaching the tufts. The nozzle diameter was larger than the tuft length, and it was ensured that the entire tuft was located inside the potential core of the steady jet. Several tufts were tested for each material, which enabled calculating both the mean deflection angle and the relative scatter for each tuft, indicated as error bars. Even though tufts were nominally oriented perpendicular to the nozzle axis in the absence of the jet $\left(U_{\infty}=0 \mathrm{~m} / \mathrm{s}\right)$, small offset angles occurred

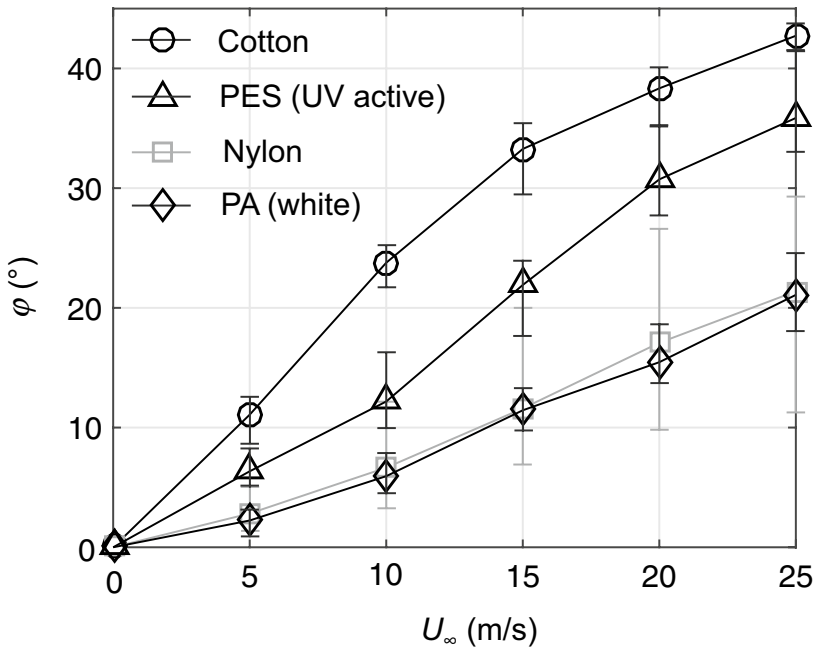

Fig. 3 Tuft deflection angle as a function of velocity for different tuft materials

occasionally. These were determined with the introduced method and subtracted from the deflection angles measured subsequently.

Due to their greater stiffness, the smallest deflection angles are found for polyamide (PA) and nylon. However, the range of deflection angles is much greater for nylon where the variance at $U_{\infty}=20 \mathrm{~m} / \mathrm{s}$ is $98 \%$ of the mean value $\varphi \approx 17.1^{\circ}$. The relative scatter for PA is $32 \%$ at the same inflow velocity. The polyester (PES) tufts exhibit a similar total variance of up to $\Delta \varphi=10^{\circ}$ but the relative variation is smaller due to greater overall deflection angles ( $24 \%$ at $\left.U_{\infty}=20 \mathrm{~m} / \mathrm{s}\right)$. The same is true for the cotton tufts that are associated with the largest deflection angles, yielding a relative deviation of $12 \%$ at $U_{\infty}=20 \mathrm{~m} / \mathrm{s}$. Even though this resembles the best reproducibility of tuft materials assessed in this study, the value is still inferior compared to other quantitative measurement techniques. The relatively low degree of reproducibility can be attributed to a number of causes which must be addressed in more detail in future studies. First, the tufts used in this study are not geometrically identical, mainly due to slight predeformations induced when handling and cutting them. Furthermore, manufacturing imperfections affect the tuft properties in the case of braided tufts. In addition to that, they were affixed with adhesive tape and the identical orientation cannot be ensured in the process. However, varying tuft characteristics can be compensated by establishing different calibration laws for individual tufts. This was done for the second demonstration case presented in Sect. 4.

Among the assessed materials, cotton tufts exhibit the most favourable characteristics and will be employed in the following. 


\subsection{Dynamic behavior of tufts}

Since a steady approach flow only reflects the conditions of a limited number of flow configurations, further generic tests were conducted to address the dynamic behavior of tufts exposed to unsteady flow. For this purpose, a pulsed jet actuator consisting of a solenoid valve and a nozzle was employed and starting jets were emitted at constant frequencies. Figure 4 shows the time signal of the deflection angle of a cotton tuft of $l=5 \mathrm{~mm}$ (open circles) for a frequency of $f=50 \mathrm{~Hz}$ and the velocity signal measured with a hot wire sensor as a reference method. The tuft is clearly capable of following the dominant frequency. This was observed to be the case for frequencies up to $f=80 \mathrm{~Hz}$. Therefore, a dynamic response required for many low-speed applications can be attested. However, it is worth pointing out that this generic test configuration does not reflect highly turbulent flows with flow features corresponding to large frequency bands. The suitability for these cases may be addressed in future applications.

\section{Demonstration of applicability}

Two test cases were investigated with the proposed measurement technique to demonstrate its capabilities: (1) the nearwall flow along a turbulent separation bubble (TSB) and (2) the wake of a generic car model.

\subsection{Test case 1: turbulent separation bubble}

TSBs are generated when a turbulent boundary layer separates from the wall and reattaches further downstream, enclosing the separation zone. The flow separation can

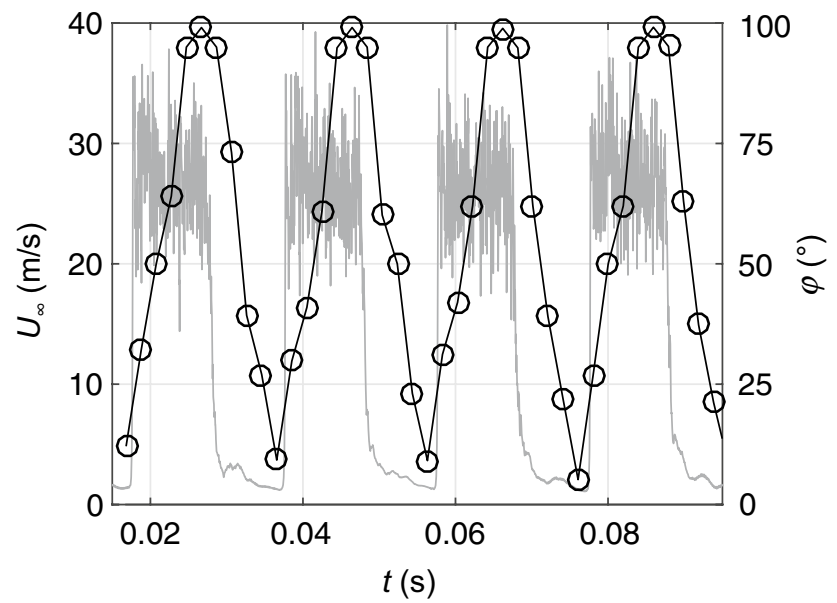

Fig. 4 Dynamic behavior of tuft subjected to $f=50 \mathrm{~Hz}$ pulsed jet, hot wire signal shown in gray color for reference either be induced by a geometric singularity, such as a backward facing step (e.g., Driver et al. 1987; Ma and Schröder 2017) or fence (e.g., Ruderich and Fernholz 1986; Hudy and Naguib 2003) or by an adverse pressure gradient (APG). In this test case, a pressure-induced TSB was investigated where the APG was introduced by the widening of the test section cross area. A similar flow configuration was investigated in Weiss et al. (2015) and Mohammed-Taifour and Weiss (2016) on the basis of unsteady pressure, HWA and PIV measurements.

In the current study, the free stream velocity was $U_{\infty}=20 \mathrm{~m} / \mathrm{s}$. The mean flow field of the TSB addressed in this study is shown in Fig. 5. The time-averaged recirculation zone associated with the TSB is enclosed by the separating streamline $\bar{u}=0 \mathrm{~m} / \mathrm{s}$, spanning a region of roughly $x \approx 0.1 \ldots 0.45 \mathrm{~m}$.

Cotton tufts $(l=15 \mathrm{~mm})$ were attached to the test section surface inside the symmetry plane with a spacing of $\Delta s=25 \mathrm{~mm}$ as indicated in the sample photography shown in Fig. 5. A second line of tufts with free ends pointing in the other direction was used for reference. While they showed good agreement with tufts placed on the symmetry plane, we will not address them in much detail here. The main
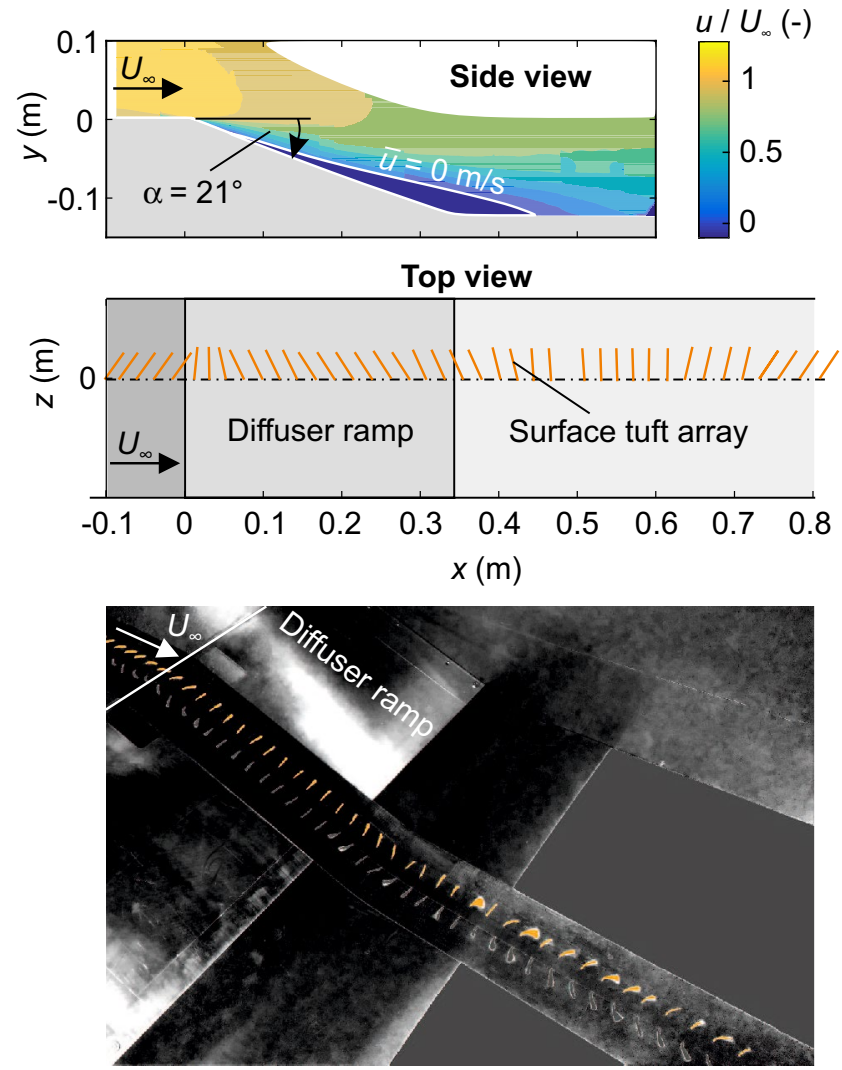

Fig. 5 TSB studied by means of TDV; time-averaged streamwise velocity field measured with PIV (top), application of surface tufts in symmetry plane (bottom) 
objective in this scenario was to identify the instantaneous flow direction and detect unsteady regions of reverse flow in particular. Therefore, the tufts were attached perpendicular to the main flow direction. Images of the tufts were taken at an acquisition rate of $f=200 \mathrm{~Hz}$ over a time duration of $\Delta t=10 \mathrm{~s}$. Due to the size of the relevant flow region, the tuft array was divided into four segments of similar length of which images were taken subsequently. To compensate the tilted diffuser ramp surface, the camera was adjusted so that the optical axis was parallel to the wall normal for all segments.

With initial tuft angles being transverse to the free stream direction, deflection angles of $\varphi>0^{\circ}$ are expected for regions of forward flow, whereas flow recirculation yields angles of $\varphi<0^{\circ}$. It is worth mentioning that the locations of separation and reattachment are not constant but fluctuate significantly. This is explained by the finding that TSBs are characterized by a low-frequency contraction and expansion in addition to medium-frequency oscillations associated with vortex structures inside the separated shear layer and highfrequency fluctuations caused by the turbulent nature of the flow (Mohammed-Taifour and Weiss 2016). Therefore, the test case is suited to assess the capability of the TDV method to measure unsteady flow conditions in close wall proximity.

Figure 6 shows the time-averaged deflection angles of tufts along the diffuser axis that are based on the time signals of tuft deflection angles obtained with the procedure introduced in Sect. 2.

As expected, the region upstream of the diffuser ramp $(x<0 \mathrm{~m})$ is characterized by positive deflection angles of $\varphi>15^{\circ}$ corresponding to the forward-flow boundary layer. Further downstream, the flow decelerates and the tuft deflection angles decrease accordingly. A mean value of $\bar{\varphi} \approx 0^{\circ}$, i.e., an average tuft orientation perpendicular to the diffuser axis, is found at $x \approx 0.15 \mathrm{~m}$ while negative deflection angles

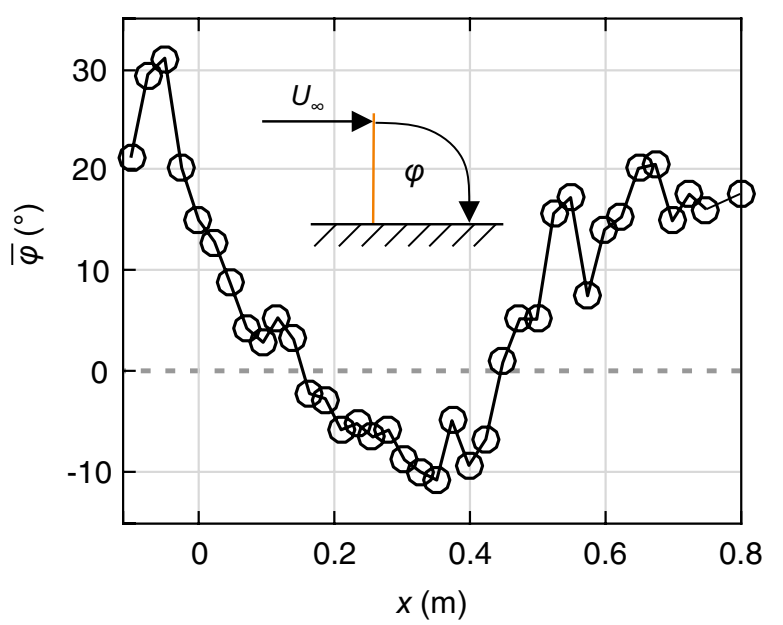

Fig. 6 Mean tuft deflection angles along diffuser axis are observed in the range of $x=0.15 \ldots 0.45 \mathrm{~m}$ due to flow recirculation in this region. This corresponds reasonably well with the mean separation line, extracted from PIV measurements, that is shown in Fig. 5. Downstream of the TSB, vortex structures are shed onto the wall and positive deflection angles are observed.

Being a characteristic quantity for the dimensions of TSBs, the forward flow coefficient $\gamma(x)$ was deduced. This quantity represents the time period during which the flow is oriented in streamwise direction $(u>0 \mathrm{~m} / \mathrm{s})$ relative to the total observation time (Simpson 1996). Here, it was approximated by the number of samples where $\varphi>0^{\circ}$ relative to the total number of samples (Fig. 7).

The evaluation of tuft angles yields two regions of $\gamma>99 \%$, being $x \leq 0 \mathrm{~m}$ and $x \geq 0.5 \mathrm{~m}$. The respective boundaries mark the positions of incipient detachment and complete reattachment as defined by Simpson (1996). The corresponding length of the enclosed TSB is $L_{99} \approx 0.5 \mathrm{~m}$ which is about $20 \%$ smaller than the value measured with PIV where the complete reattachment point was not inside the measurement domain but a location of $x \approx 0.6 \mathrm{~m}$ can be assumed. The main reason for this deviation is the difference in forward flow coefficients at the downstream end of the TSB. Whereas PIV measurements reveal a number of instantaneous snapshots where recirculation occurs, this information is not captured by TDV. The negative streamwise velocity component is induced by vortex structures originating from the separated shear layer and the time duration may be too short to cause negative deflection angles. However, smaller tuft dimensions may be able to render this process more accurately. The same effect is present inside the TSB

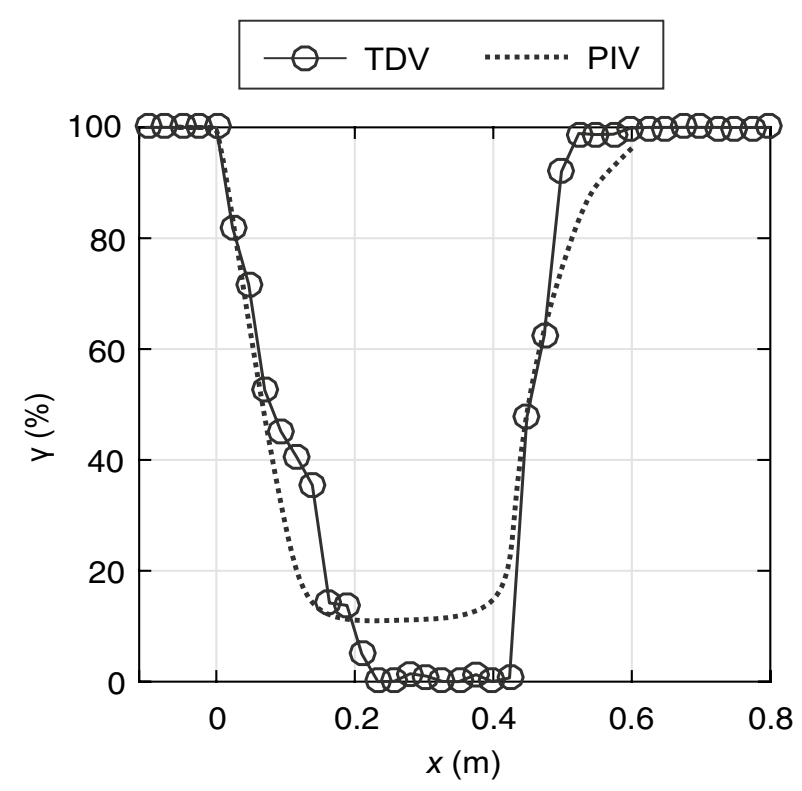

Fig. 7 Forward flow ratio along diffuser axis determined by means of TDV and PIV, respectively 
where PIV measurements suggest forward flow coefficients of $\gamma_{\text {PIV }} \approx 15 \%$ whereas tuft deflection angles are negative at almost all times, hence $\gamma_{\mathrm{TDV}} \approx 0 \%$. However, the locations of transitory detachment and reattachment marked by $\gamma=50 \%$ are well captured by the technique introduced in this article, and a TSB length of $L_{50} \approx 0.38 \mathrm{~m}$ is derived both from PIV and TDV measurements. It should be noted, however, that the location of transitory detachment as derived from the forward flow ratio is slightly upstream of the location where $\bar{\varphi}=0^{\circ}$. This can be explained by the fact that close to the location of transitory detachment, the magnitude of deflection angles is larger in the case of forward-flow even though the respective time duration is equal to that of reverse flow. Since this was not observed in PIV measurements, we attribute this finding to individual tufts having a preferential bending direction.

Finally, the fluctuation level of tuft deflection angles is evaluated along the TSB. As a reference, unsteady pressure measurements are conducted with piezo-resistive pressure transducers. Both distributions are shown in Fig. 8. In good agreement with results in the literature (Mohammed-Taifour and Weiss 2016), two local peaks are found close to the mean locations of separation and reattachment. This is also reflected by the TDV analysis where maxima of the order of $\operatorname{rms}\left(\varphi^{\prime}\right) \approx 6^{\circ}$ and $\operatorname{rms}\left(\varphi^{\prime}\right) \approx 13^{\circ}$ are found, respectively.

Generally, the first test case shows that the method is well suited to adequately detect the instantaneous flow direction when applied in the near-wall region. Furthermore, an analysis of unsteady flow was successfully conducted to determine regions of maximum fluctuation level inside a flow configuration. The frequency peak associated with these fluctuations was in the region of $f \approx 60 \ldots 80 \mathrm{~Hz}$. However,

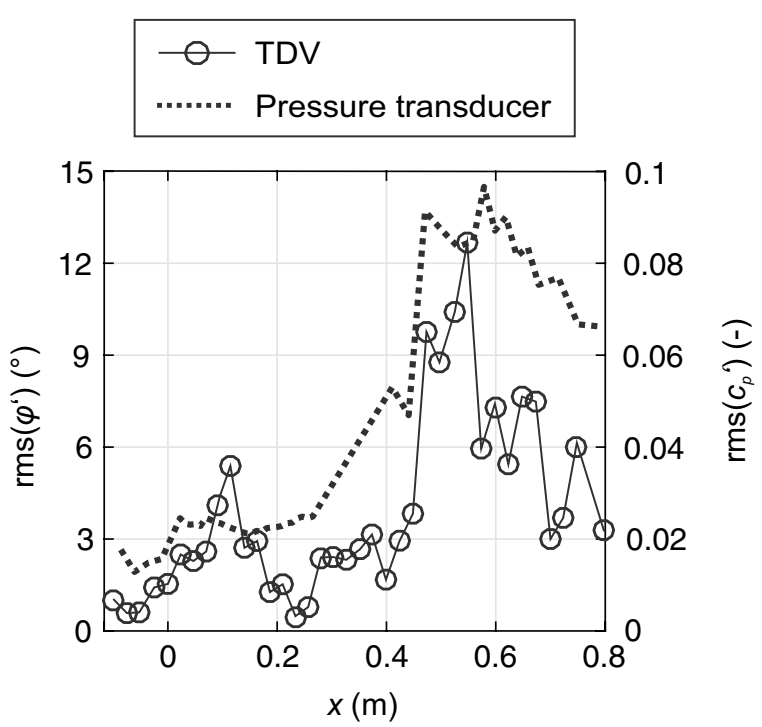

Fig. 8 Fluctuation level along diffuser axis determined by means of TDV and piezo-resistive pressure transducer, respectively errors occur when the flow is dominated by higher-frequency flow reversals.

\subsection{Test case 2: wake of generic car model}

A second test configuration was chosen to assess the performance of the TDV method when a free velocity field is analyzed, i.e., when the tufts are not attached to a solid surface. For this purpose, an Ahmed body - a generic car geometry introduced in Ahmed et al. (1984)—was studied inside the same low-speed wind tunnel as the TSB (test case 1) at a free stream velocity of $U_{\infty}=20 \mathrm{~m} / \mathrm{s}$. Based on the height of the $50 \%$ scale model $H \approx 0.14 \mathrm{~m}$, the Reynolds number was $R e_{\mathrm{H}} \approx 1.9 \cdot 10^{5}$. The wake topology subject to a varied slant inclination angle $\beta$ is well known from previous studies (Ahmed et al. 1984). The smallest drag coefficient is found at $\beta \approx 10^{\circ}$ where the flow is attached on the slant surface. The drag then increases for greater inclination angles due to the enhancement of streamwise vortices originating at the c-pillars. At a slant angle of $\beta \approx 30^{\circ}$, these vortices break down and a discontinuous drop in drag occurs. While the large-scale streamwise vortices will not be addressed in this study, we employed TDV to investigate the influence of the slant inclination on the dimensions of the recirculation zone inside the symmetry plane. The setup for this test case is schematically depicted in Fig. 9. Two slant angles, $\beta=15^{\circ}$ and $\beta=35^{\circ}$, were investigated and a probe with 50 cotton tufts of $l=5 \mathrm{~mm}$ length was traversed in the symmetry plane in $\Delta x=4 \mathrm{~mm}$ steps along the $x$ direction between $x=-100 \ldots 200 \mathrm{~mm}$ where $x=0 \mathrm{~mm}$ marks the base of the Ahmed body. The probe consisted of a solid beam with drilled holes through which the tufts were sticked and glued on the back side of the probe. The vertical spacing between neighboring tufts was $\Delta y=4 \mathrm{~mm}$. The optical axis of the camera was normal on the symmetry plane (Fig. 9), and images were taken over a duration of $\Delta t=3 \mathrm{~s}$ with an

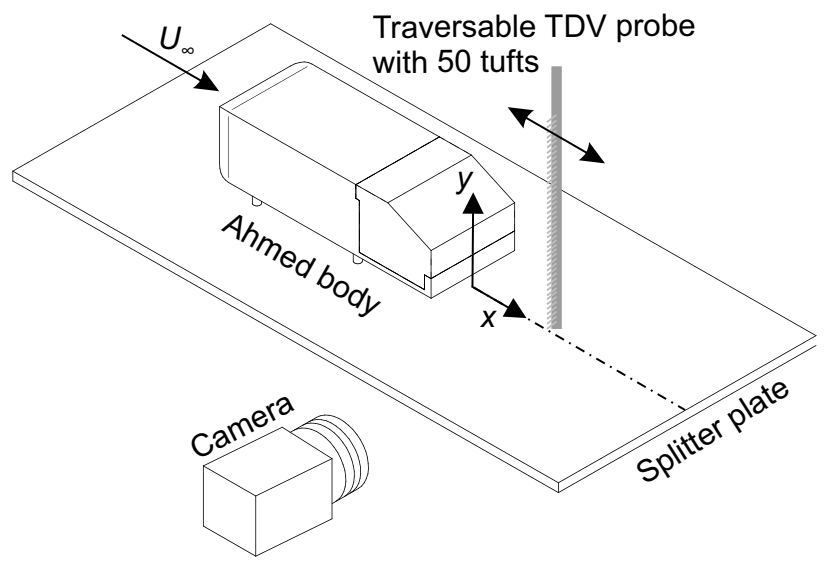

Fig. 9 Setup for flow field measurement of Ahmed body wake by means of TDV 
acquisition rate of $f=400 \mathrm{~Hz}$. This was sufficient to resolve relevant shedding modes that are associated with a Strouhal number of $S t \approx 0.22$ at $\beta=35^{\circ}$ according to Tunay et al. (2014), corresponding to a frequency of $f \approx 30 \mathrm{~Hz}$.

Measurements were conducted inside the symmetry plane where the out-of-plane component is assumedly negligible. The initial tuft orientation was parallel to the $z$ axis, and the tufts were free to move in two directions, namely $x$ and $y$ in this application scenario. The evaluation algorithm was therefore extended to compute two separate deflection angles corresponding to both in-plane velocity components in the symmetry plane. As indicated above, the initial orientation of the tufts was almost parallel with regard to the optical axis, resulting in almost zero length when imaged by the camera. The projected length of the tufts was used to obtain deflection angles. Again, 2D2C PIV measurements were employed as a reference measurement technique. Here, mean values of the two velocity components were obtained based on 500 incoherent snapshots. The distance between vectors measured with PIV was $\Delta x=\Delta y \approx 1 \mathrm{~mm}$, yielding a four times finer resolution than for TDV measurements.

Since the preliminary analysis showed that the characteristic tuft deflection curve is not identical for different tufts, an initial calibration was performed by applying different flow velocities relevant to this test case and measuring the individual deflection angles in the absence of the Ahmed body. The characteristics of individual tufts were then mapped with second-order polynomials shown in Fig. 10. Since the cotton tufts used for this test case were shorter than those addressed in Fig. 3, the deflection angles are smaller at equal velocities. The two in-plane velocity components $u$ and $v$ were computed based on the individual calibrations where the free stream velocity was varied in

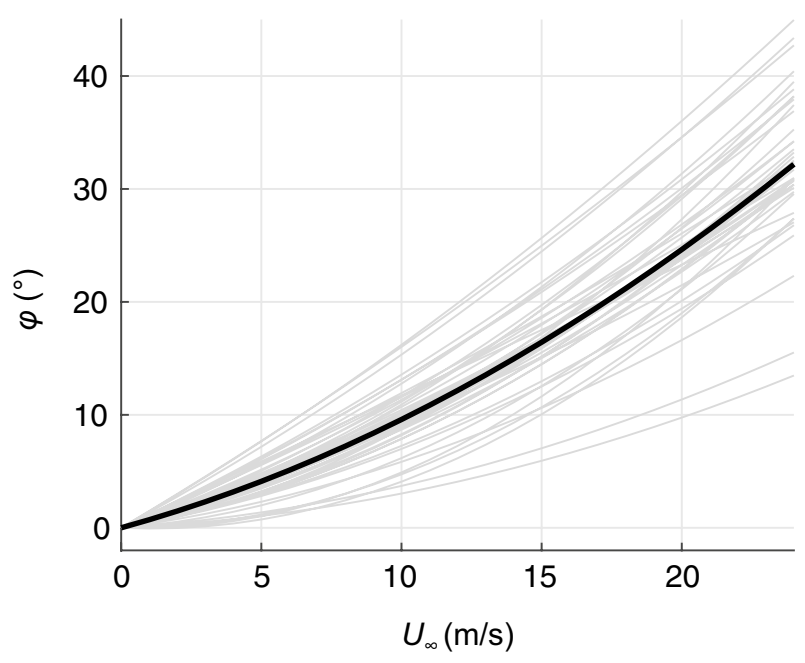

Fig. 10 Second-order polynomials for velocity calibration of individual tufts, mean value printed bold the range $u=0 \ldots 25 \mathrm{~m} / \mathrm{s}$ (Fig. 10). Identical characteristics were assumed for negative velocities in main stream direction and for the vertical velocity component. In hindsight, this assumption is probably not justified as a preferential direction of the tufts was indicated by results of the previous test case where different deflection angles occur for the same forcing.

Generally, the additional effort required for this calibration was minimal since no modification to the experimental setup was needed in terms of the optical arrangement.

The main results of this experiment are shown in Fig. 11 where the contour field of the normalized streamwise velocity component is overlayed with the time-averaged streamline pattern that is based on the measured vector fields.

The longitudinal extent of the detected recirculation zones measured with TDV agrees well with the length obtained with PIV measurements. The stagnation point is located at $x \approx 150 \mathrm{~mm}$ for the $\beta=15^{\circ}$ slant angle whereas a longer recirculation zone is found for $\beta=35^{\circ}$ where the stagnation point is at $x \approx 180 \mathrm{~mm}$. Furthermore, the upper part of the vortex ring rooted at the base is captured by the method. However, there is a large difference in the velocity magnitude inside the recirculation zone determined by both methods. The approach introduced in this article suggests a minimum longitudinal velocity of $u \approx-0.6 U_{\infty}$ compared to $u \approx-0.4 U_{\infty}$ obtained from PIV measurements. In general, there is a systematic difference between both measurements of the order of $\Delta u=\left(u_{\mathrm{PIV}}-u_{\mathrm{TDV}}\right) / U_{\infty}>0.1$ inside the recirculation zone. A significant deviation of opposite sign in terms of the streamwise velocity inside the shear layers bounding the recirculation zone is also observed. This may be attributed to the small velocity magnitude $|u| \rightarrow 0 \mathrm{~m} / \mathrm{s}$ present in these regions. As a result of this inaccuracy, the recirculation zone measured with TDV is systematically smaller in vertical direction.

Nonetheless, the second test case demonstrates that the TDV technique can be employed to gain a quantitative impression of a 2D2C flow field with a measurement setup that is much easier, cheaper and simpler to establish compared to PIV measurements. The measurement accuracy may be adequate for a preliminary assessment of complex flows, though it is probably inacceptable for detailed analyses. This insufficiency is mainly linked with the material properties of the cotton tufts used in this study.

\section{Conclusions}

The main objective of this article was to introduce a novel measurement method that allows for a quick and simple quantitative analysis of flow fields. Two test configurations have demonstrated the potential of this approach. However, the current limitations preclude a replacement of 


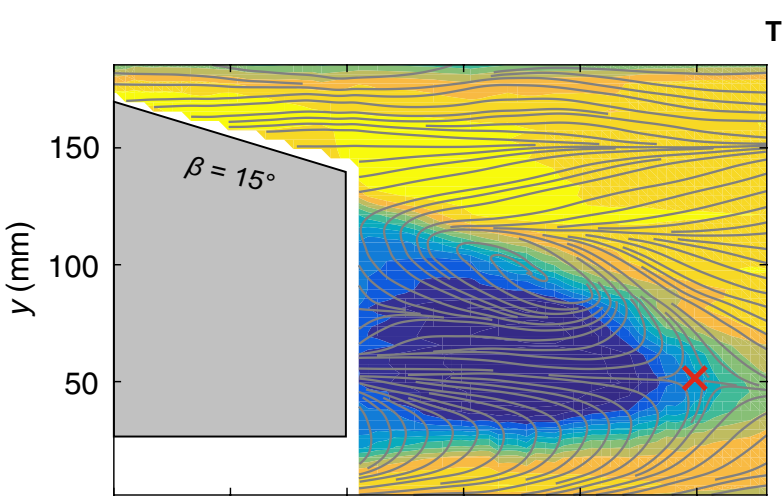

TDV

$u / U_{\infty}(-)$
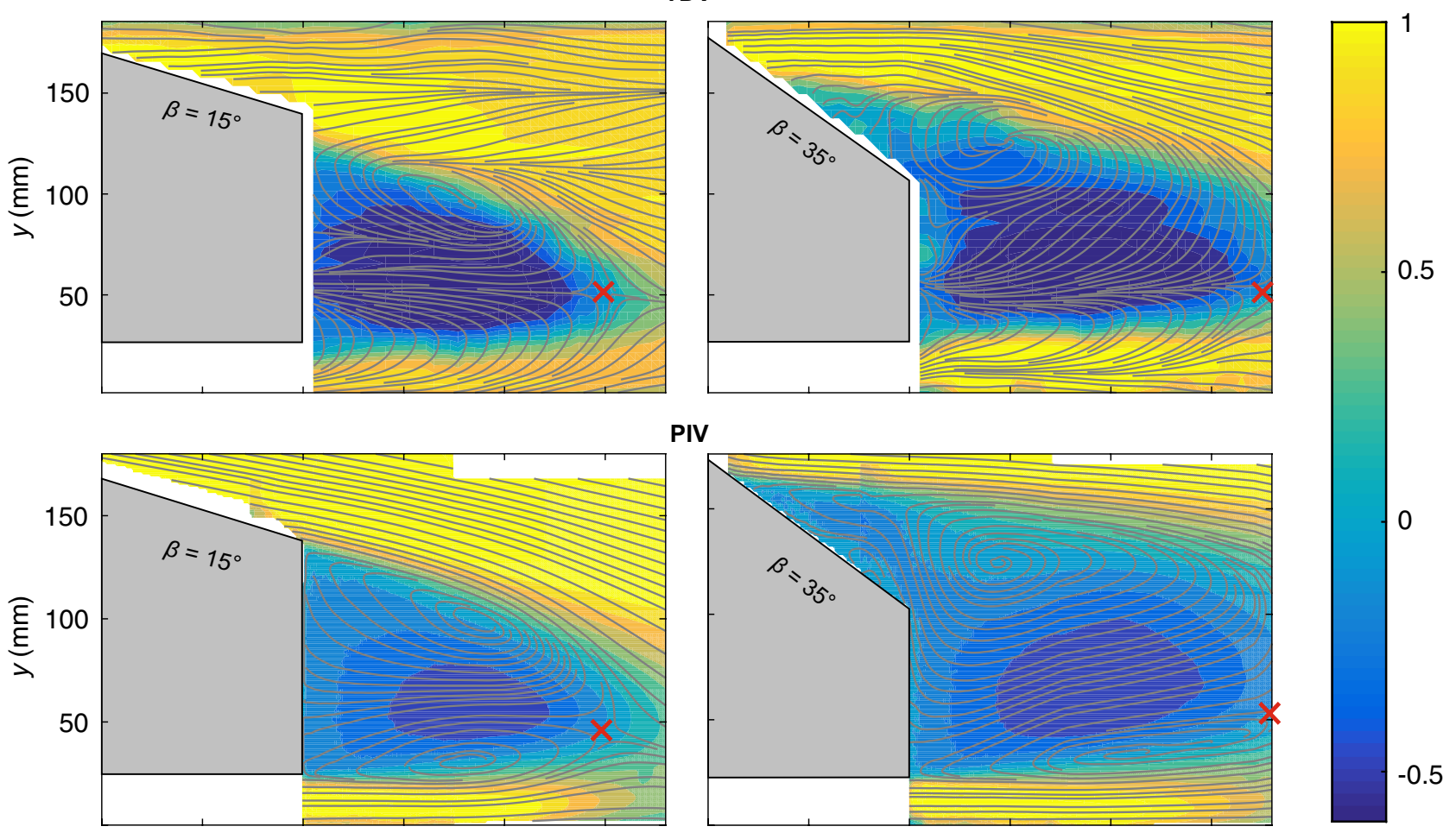

PIV
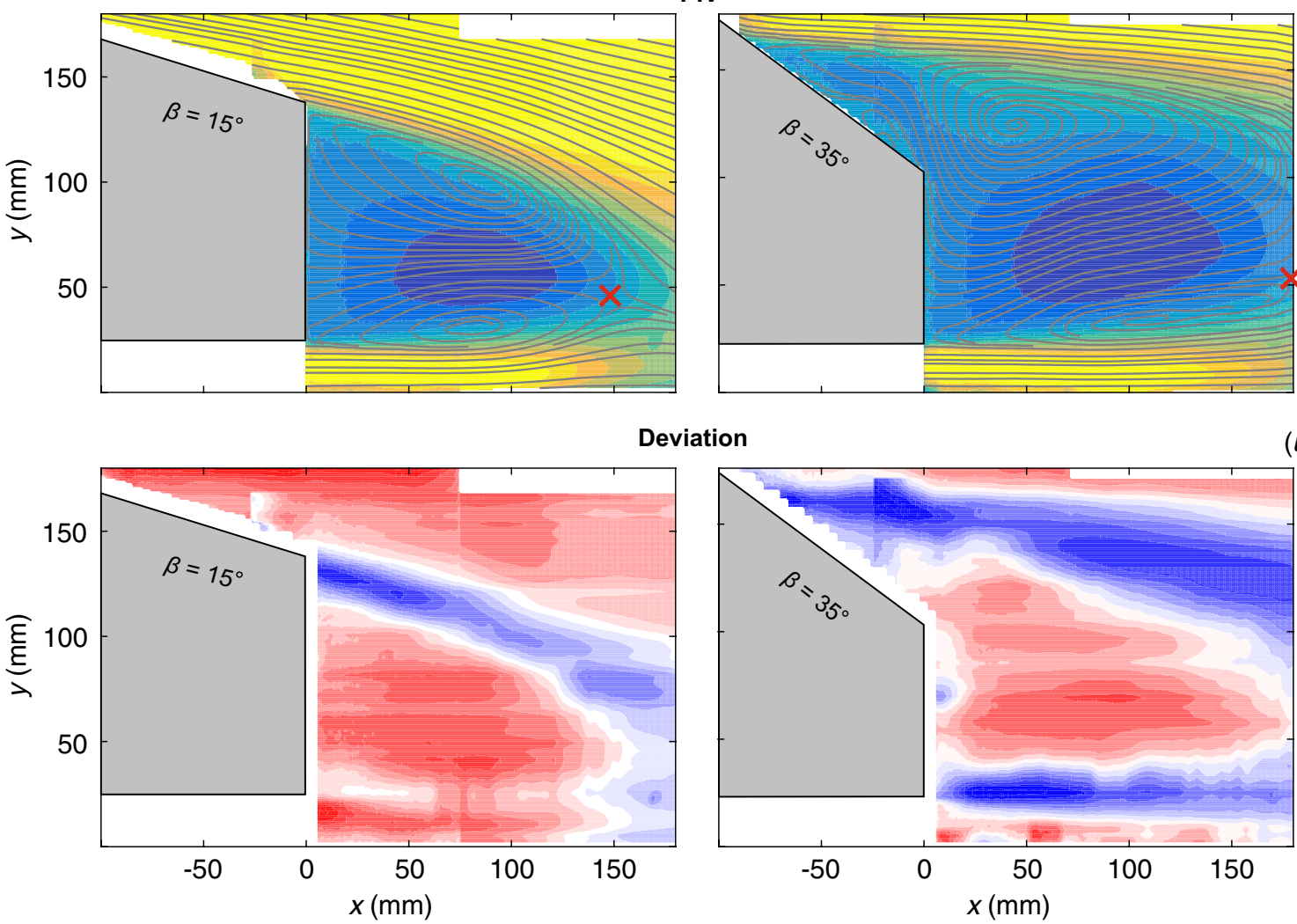

$\left(u_{\mathrm{PIV}}-u_{\mathrm{TDV}}\right) / U_{\infty}(-)$

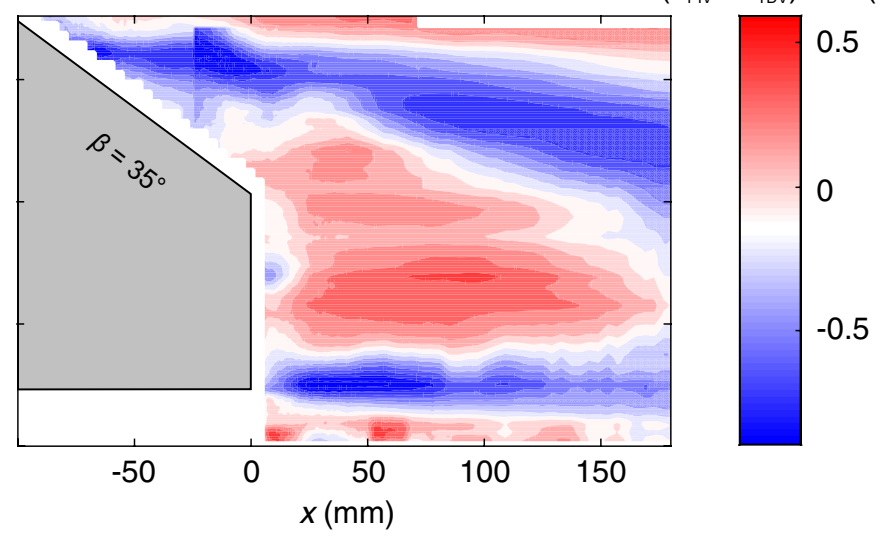

Fig. 11 Velocity field in the wake of an Ahmed body; measured with TDV (top row) and PIV (center row) and deviation between velocity fields obtained with both methods (bottom row); stagnation point marked with red cross

conventional measurement techniques. We therefore hope that the approach is adapted and modified by other researchers, gradually optimizing the method as has been done with other measurement techniques in the past.

\subsection{Advantages and disadvantages}

The main advantage of TDV lies in its quick and simple implementation. No elaborate measurement equipment is required and the data processing is fast compared to other techniques for flow field measurements. A camera is on hand in practically all research facilities, and the data analysis can be performed on a standard work station. In addition to that, the method can be executed by new users after a very short initial training phase due to the intuitive function principle. At the same time, the risk of committing fatal errors with consequences for the health of the user or damages of equipment during that training phase is very low. As another main advantage, information regarding large flow fields can be obtained in a relatively short amount of time compared to pointwise measurement techniques and also PIV where the measurement domain is 
typically smaller due to a limited region of sufficient laser light energy and a stronger reliance on optical resolution to render seeding particles.

These potential benefits are currently offset by an insufficient measurement accuracy that is mainly due to a very limited reproducibility of the tuft characteristics. While not directly addressed in this article, TDV is an intrusive method and the user must be aware that required instrumentation, e.g., probes and tufts, may have a significant influence on the flow field. Furthermore, tufts of one specific geometry are only suitable to a certain range of flow velocities so that an appropriate tuft material and length must be chosen according to the relevant velocity range. The results of this study have also shown that the technique may not be suited for flows dominated by features at time scales associated with frequencies of $f>100 \mathrm{~Hz}$.

As with every other experimental technique, an integration along a specific spatial domain is performed. For the TDV method, this domain is equal to the tuft which will usually have greater dimensions than the integration regions for other methods. It is therefore an inherent challenge of the TDV technique to assign measured velocity vectors to defined locations in the flow field. This difficulty is amplified when tufts are strongly bent or even twisted. As a general rule, every attempt should be made to avoid such deformations, for instance by using tufts that are as short as possible and by applying them with an adequate orientation.

\subsection{Potential fields of application}

As mentioned in the previous section, the method proposed in this article is currently not able to substitute a classical measurement technique. We therefore advocate an employment in a supplemental manner, e.g., for preliminary analyses to narrow down the parameter space for subsequent measurements.

Being a method capable of delivering information regarding large flow fields in relatively short amounts of time, TDV may nonetheless be readily utilized where PIV measurements are precluded. This may be the case when no PIV system is on hand in the first place or when PIV cannot be employed for safety reasons, e.g., outdoors or when contamination with seeding particles is not desired. Furthermore, TDV is conceptually not restricted to two-dimensional measurement domains as tufts may be attached to curved surfaces with images readily dewarped if required.

Generally, there is a very large number of potential application scenarios, including near-wall measurements but also an employment inside wall-distant flows, and we hope that this will be reflected in future studies by other research groups.

\subsection{Outlook}

Future efforts must be primarily aimed at improving the measurement accuracy of the method proposed here. From our point of view, this can be achieved by identifying a tuft material with more reproducible properties. In the current study, cotton tufts were utilized, exhibiting drastically varying mechanical properties despite their geometric similarity. We also observed that occasionally, the characteristic deflection behavior changed over time as irreversible deformation of the tufts occurred after being exposed to the flow impulse. We therefore firmly believe that significant improvement can be brought about by identifying a more appropriate tuft material.

Acknowledgements Open Access funding provided by Projekt DEAL. The authors gratefully acknowledge the assistance from Steffen Feldhus and Yannick Kather with wind tunnel measurements for the first demonstration case.

Code availability A minimum working example of the algorithm introduced in this article is provided with the supplementary material.

\section{Compliance with ethical standards}

Conflict of interest The authors declare that they have no conflict of interest.

Open Access This article is licensed under a Creative Commons Attribution 4.0 International License, which permits use, sharing, adaptation, distribution and reproduction in any medium or format, as long as you give appropriate credit to the original author(s) and the source, provide a link to the Creative Commons licence, and indicate if changes were made. The images or other third party material in this article are included in the article's Creative Commons licence, unless indicated otherwise in a credit line to the material. If material is not included in the article's Creative Commons licence and your intended use is not permitted by statutory regulation or exceeds the permitted use, you will need to obtain permission directly from the copyright holder. To view a copy of this licence, visit http://creativecommons.org/licenses/by/4.0/.

\section{References}

Ahmed SR, Ramm G, Faltin G (1984) Some salient features of the time-averaged ground vehicle wake. SAE Trans 93:473-503

Andino MY, Lin JC, Washburn AE, Whalen EA, Graff EC, Wygnanski IJ (2015) Flow separation control on a full-scale vertical tail model using sweeping jet actuators. In: 53rd AIAA aerospace sciences meeting, p 0785

Bird JD, Riley DR (1952) Some experiments on visualization of flow fields behind low-aspect-ratio wings by means of a tuft grid. Technical report, National Advisory Committee for Aeronautics (NASA)

Brown GL, Roshko A (1974) On density effects and large structure in turbulent mixing layers. J Fluid Mech 64(4):775-816

Chen L, Suzuki T, Nonomura T, Asai K (2019) Characterization of luminescent mini-tufts in quantitative flow visualization 
experiments: surface flow analysis and modelization. Exp Therm Fluid Sci 103:406-417

Chen L, Suzuki T, Nonomura T, Asai K (2020) Flow visualization and transient behavior analysis of luminescent mini-tufts after a backward-facing step. Flow Meas Instrum 71:101,657

Driver DM, Seegmiller HL, Marvin JG (1987) Time-dependent behavior of a reattaching shear layer. AIAA J 25(7):914-919. https://doi.org/10.2514/3.9722

Duda RO, Hart PE (1972) Use of the Hough transformation to detect lines and curves in pictures. Commun ACM 15(1):11-15. https ://doi.org/10.1145/361237.361242

Eggleston DM, Starcher K (1990) A comparative study of the aerodynamics of several wind turbines using flow visualization. $\mathrm{J}$ Sol Energy Eng 112(4):301-309

Fisher DF, DelFrate JH, Richwine DM (1991) In-flight flow visualization characteristics of the NASA F-18 high alpha research vehicle at high angles of attack. Technical report, NASA Dryden Flight Research Facility

Hudy LM, Naguib AM (2003) Wall-pressure-array measurements beneath a separating/reattaching flow region. Phys Fluids. https ://doi.org/10.1063/1.1540633

Ma X, Schröder A (2017) Analysis of flapping motion of reattaching shear layer behind a two-dimensional backward-facing step. Phys Fluids. https://doi.org/10.1063/1.4996622

Mason W, Marchman III J (1971) Investigation of an aircraft trailing vortex using a tuft grid. Technical report, Virginia Polytechnic Institute, Department of Aerospace Engineering

McCormick BW, Sherrier H, Tangler J (1968) Structure of trailing vortices. J Aircr 5(3):260-267

Mohammed-Taifour A, Weiss J (2016) Unsteadiness in a large turbulent separation bubble. J Fluid Mech. https://doi.org/10.1017/ jfm.2016.377

Prewitt JMS (1970) Object enhancement and extractionl. In: Lipkin B, Rosenfeld A (eds) Picture processing and psychopictorics. Academic Press, New York, pp 75-149

Ristić S (2007) Flow visualisation techniques in wind tunnels part Inon optical methods. Sci Tech Rev 57(1):39-50
Ruderich R, Fernholz HH (1986) An experimental investigation of a turbulent shear flow with separation, reverse flow, and reattachment. J Fluid Mech 163:283-322. https://doi.org/10.1017/S0022 112086002306

Simpson RL (1996) Aspects of turbulent boundary-layer separation. Prog Aerosp Sci 32(5):457-521. https://doi.org/10.1016/03760421(95)00012-7

Steinfurth B, Berthold A, Feldhus S, Haucke F, Weiss J (2019) Increasing the aerodynamic performance of a formula student race car by means of active flow control. SAE Int J Adv Curr Pract Mobil. https://doi.org/10.4271/2019-01-0652

Swytink-Binnema N, Johnson DA (2016) Digital tuft analysis of stall on operational wind turbines. Wind Energy 19(4):703-715

Tunay T, Sahin B, Ozbolat V (2014) Effects of rear slant angles on the flow characteristics of ahmed body. Exp Therm Fluid Sci 57:165-176. https://doi.org/10.1016/j.expthermflusci.2014.04.016

Vey S, Lang HM, Nayeri CN, Paschereit CO, Pechlivanoglou G (2014) Extracting quantitative data from tuft flow visualizations on utility scale wind turbines. J Phys Conf Ser 524:012011

Weiss J, Mohammed-Taifour A, Schwaab Q (2015) Unsteady behavior of a pressure-induced turbulent separation bubble. AIAA J 53(9):2634-2645. https://doi.org/10.2514/1.J053778

Werle H (1973) Hydrodynamic flow visualization. Annu Rev Fluid Mech 5(1):361-386

Wieser D, Bonitz S, Löfdahl L, Broniewisz A, Nayeri CN, Paschereit CO, Larsson L (2016) Surface flow visualization on a full-scale passenger car with quantitative tuft image processing. SAE Technical Paper 2016-01-1582. https://doi.org/10.4271/2016-01-1582

Wieser D, Lang H, Nayeri C, Paschereit C (2015) Manipulation of the aerodynamic behavior of the drivAer model with fluidic oscillators. SAE Int J Passeng Cars-Mech Syst 8:687-702

Publisher's Note Springer Nature remains neutral with regard to jurisdictional claims in published maps and institutional affiliations. 